Polymer Journal, Vol. 38, No. 10, pp. 1055-1060 (2006)

(C) 2006 The Society of Polymer Science, Japan

\title{
Highly Isotactic Poly(vinyl alcohol) Derived from tert-Butyl Vinyl Ether V. Viscoelastic Behavior of Highly Isotactic Poly(vinyl alcohol) Films
}

\author{
Hiroyuki OHgi, ${ }^{1, \dagger}$ Toshiaki SATo, ${ }^{1}$ Hiroshi WATAnABE, ${ }^{2}$ and Fumitaka HorII ${ }^{2}$ \\ ${ }^{1}$ Tsukuba Research Laboratories, Kuraray Co., LTD., 41, Miyukigaoka, Tsukuba 305-0841, Japan \\ ${ }^{2}$ Institute for Chemical Research, Kyoto University, Uji, Kyoto 611-0011, Japan
}

(Received February 21, 2006; Accepted July 25, 2006; Published September 7, 2006)

\begin{abstract}
Dynamic viscoeasticity of poly(vinyl alcohol) with high isotacticity (HI-PVA, $m m=0.78$ ), being derived from tert-butyl vinyl ether, was investigated to compare with that of syndiotactc PVA (S-PVA, $m m=0.14)$ and commercial atactic PVA (A-PVA, $m m=0.22$ ). In the non-crystalline region, the $\beta_{\mathrm{a}}$ (local twisting motion) and $\alpha_{\mathrm{a}}$ (micro-Brownian motion) dispersions occurring at around $-10^{\circ} \mathrm{C}$ and $70^{\circ} \mathrm{C}$ were nearly the same, in both magnitude and location, for the HI-PVA and S-PVA having an almost identical degree of crystallinity. On the other hand, in the crystalline region, the $\beta_{\mathrm{c}}$ and $\alpha_{\mathrm{c}}$ dispersions of HI-PVA were somewhat different from those of ordinary PVAs. The $\beta_{\mathrm{c}}$ dispersion (local motion in crystals due to defects) was clearly observed for HI-PVA but not for S-PVA and A-PVA. The $\alpha_{\mathrm{c}}$ dispersion (axial motion of the chain in the crystal lattices) was observed for all PVA films but its temperature increased in the order of HI-PVA $>$ S-PVA $>$ A-PVA, which well corresponded to the order of the melting temperature and ${ }^{13} \mathrm{C}$ spin-lattice relaxation time $\left(T_{1 \mathrm{C}}\right)$ of each stereoregular PVA. These differences may be attributed to a difference of the magnitude of intermolecular and/or intramolecular hydrogen bonding in the crystals of respective PVAs. Specifically, the successive intramolecular bonding in the HI-PVA crystal appears to reduce the magnitude of the intermolecular bonding thereby allowing the chain to exhibit the $\beta_{\mathrm{c}}$ motion. This successive bonding would also stiffen the chain backbone thereby increasing the $\alpha_{\mathrm{c}}$ dispersion temperature. [doi:10.1295/polymj.PJ2005235]

KEY WORDS Highly Isotactic Poly(vinyl alcohol) / Stereoregular Polymerization /

Viscoelasticity /
\end{abstract}

Many efforts have been made for synthesis of stereoregular poly(vinyl alcohol)s (PVAs) being rich in syndiotactic or isotactic sequences ${ }^{1-3}$ and changes of their physical properties with the stereoregularity, possibly reflecting changes in the inter- and intramolecular hydrogen bonding, have been extensively investigated. A series of radical-polymerized poly(vinyl ester)s was utilized to synthesize syndiotactic PVAs, and the relationships between stereoregularity and physical properties of PVA have been examined. ${ }^{1-3}$ Isotactic PVAs were synthesized successfully from poly(vinyl ether)s such as poly(benzyl vinyl ether) (PBzVE), ${ }^{4,5}$ poly(tert-butyl vinyl ether) (PtBVE), ${ }^{6,7}$ and poly(trimethyl silyl vinyl ether) (PVOSi), ${ }^{8,9}$ and their physical properties were also investigated. ${ }^{1,3}$

Despite the above efforts, the stereoregularity still remained at a low level of the $\mathrm{mm}$ fraction $(=0.70)$ even for so-called isotactic PVA (LI-PVA) derived from PVOSi. ${ }^{9}{ }^{10}$ Recently, we studied the cationic polymerization of $t \mathrm{BVE}$ with boron trifluoride diethyl etherate $\left(\mathrm{BF}_{3} \cdot \mathrm{OEt}_{2}\right)$ and successfully prepared the PVAs having the highest isotacticity so far reported. ${ }^{10-12}$ We found interesting features of these highly isotactic PVAs (HI-PVAs) being different from those of LI-PVA and ordinary atactic PVA (A-PVA). ${ }^{10,13,14}$ In particular, we compared the melting temperature $T_{\mathrm{m}}$, degree of crystallinity $\chi_{\mathrm{c}}$, and ${ }^{13} \mathrm{C}$ spin-lattice relaxation time $T_{1 \mathrm{C}}$ of the crystalline components for PVA samples with different tacticities (including HIPVA) to find that these physical quantities show clear minima at the $\mathrm{mm}$ fraction $=0.4-0.5$ when plotted against $\mathrm{mm} .{ }^{14}$ This result suggests that the structural disordering associated with the decrease in the crystallinity occurs most significantly in this $\mathrm{mm}$ range. In relation to these minima, FT-IR spectroscopy confirmed the formation of the new crystal form for HIPVAs with $m m>0.55$ and solid-state ${ }^{13} \mathrm{C}$ NMR spectroscopy revealed that all $\mathrm{OH}$ groups are allowed to form successive intramolecular hydrogen bonding along the respective chains in the crystalline region for HI-PVAs with $\mathrm{mm}>0.7$. On the basis of the line shape analysis of the $\mathrm{CP} / \mathrm{MAS}{ }^{13} \mathrm{C}$ NMR spectra of the crystalline components, the minima of $T_{\mathrm{m}}, \chi_{\mathrm{c}}$, and $T_{1 \mathrm{C}}$ were mainly related to the maximum fraction of disordered units introduced in the syndiotactic or isotactic sequences (that form successive inter- or intramolecular hydrogen bonding, respectively).

It is of our interest to elucidate the effect of the ster-

${ }^{\dagger}$ To whom correspondence should be addressed (Tel: +81-29-853-1521, Fax: +81-29-853-1543, E-mail: hiroyuki_ogi@kuraray.co.jp). 
eoregularity on the motion of the PVA chains in addition to the above structural features. This motion can be conveniently examined through dynamic viscoelastic behavior. Indeed, Nagai et al. ${ }^{15}$ examined this behavior for A-PVA, S-PVA and LI-PVA and found that the decrease of the dynamic Young's modulus $E^{\prime}$ (at $138 \mathrm{~Hz}$ ) at high temperatures is larger in the order, S-PVA > A-PVA > LI-PVA. However, the viscoelastic behavior of the HI-PVA with $\mathrm{mm}$ fraction higher than 0.70 has not yet been investigated, and the effect of the stereoregularity on this behavior and underlying chain motion has not been fully elucidated. Thus, we have synthesized HI-PVAs having higher $m m$ values from poly $(t \mathrm{BVE})$ and compared their behavior with that of A-PVA $(m m=0.22)$ and S-PVA $(m m=0.14)$. It turned out that the stereoregularity hardly affects the viscoelastic behavior of the noncrystalline component but a significant effect (related to the hydrogen bonding) is observed for the behavior of the crystalline component. Details of these results are reported in this paper.

\section{EXPERIMENTAL}

\section{Synthesis of Stereoregular PVAs}

HI-PVAs with $\mathrm{mm}$ triad fractions higher than 0.70 were synthesized by the following procedure: ${ }^{11}$ The $t \mathrm{BVE}$ monomer was prepared by the alkyl exchange reaction between tert-butyl alcohol and lauryl vinyl ether in the presence of $\mathrm{Hg}(\mathrm{OAc})_{2}$. Solvents were purified and dehydrated carefully by the conventional methods. Toluene was distilled in the presence of metallic sodium before use. $\mathrm{BF}_{3} \cdot \mathrm{OEt}_{2}$ was purified by distillation under reduced nitrogen pressure and used as a toluene solution. Polymerization of $t \mathrm{BVE}$ was carried out with $\mathrm{BF}_{3} \cdot \mathrm{OEt}_{2}$ in toluene at $-78^{\circ} \mathrm{C}$, and the resulting $\mathrm{P} t \mathrm{BVE}$ was converted into PVA by cleavage of the ether linkage using hydrogen bromide at $0^{\circ} \mathrm{C}$.

A commercially available A-PVA derived from poly(vinyl acetate) and syndiotactic PVA (S-PVA) derived from poly(vinyl pivalate) with the method previously reported ${ }^{16}$ were used as the reference materials to clarify unique, solid-state properties of HI-PVAs.

\section{Determination of Stereoregularity of PVAs}

The stereoregularity of PVA samples was determined from solution-state ${ }^{1} \mathrm{H}$ NMR spectroscopy on the basis of the methods ${ }^{17,18}$ reported previously. ${ }^{1} \mathrm{H}$ NMR spectra were measured in deuterated dimethyl sulfoxide (DMSO- $d_{6}$ ) at $25.0^{\circ} \mathrm{C}$ on a JEOL GSX-270 spectrometer operating at $270.17 \mathrm{MHz}$.

\section{Preparation of PVA Films}

All physical properties were measured for the PVA films with a thickness of about $100 \mu \mathrm{m}$ unless otherwise specified. The PVA films were prepared by casting $5 \mathrm{wt} \%$ DMSO solutions on glass plates, allowing DMSO to evaporate at $50^{\circ} \mathrm{C}$ for $20 \mathrm{~h}$, immersing them in hot methanol overnight, and then drying in vacuo at room temperature for $2 \mathrm{~d}$. The films were annealed at several temperatures for $30 \mathrm{~min}$.

\section{Dynamic Viscoelasticity}

Dynamic storage Young's modulus $\left(E^{\prime}\right)$, loss modulus $\left(E^{\prime \prime}\right)$ and loss tangent $(\tan \delta)$ were measured at $110 \mathrm{~Hz}$ with a heating rate of $2{ }^{\circ} \mathrm{C} / \mathrm{min}$ from $-50^{\circ} \mathrm{C}$ to $250^{\circ} \mathrm{C}$ using a dynamic viscoelastic spectrometer DVE-V4 (Rheology Engineering Co., Ltd.).

\section{Density, Swelling and DSC Tests}

The densities of PVA films prepared as above were measured with a density gradient tube (Shibayama Kagaku) using a mixture of $n$-heptane and carbon tetrachloride at $25^{\circ} \mathrm{C}$.

For the swelling experiment, the PVA films were soaked in water at room temperature for $24 \mathrm{~h}$ to be fully equilibrated in water. The degree of equilibrium swelling, $Q$, was determined by the following formula: $Q=$ (weight of swollen films - weight of dried films after swelling)/(weight of dried films after swelling).

Differential scanning calorimetry (DSC) thermograms were recorded on $10 \mathrm{mg}$ of each PVA films under nitrogen with a Mettler differential scanning calorimeter. The heat of melting $(\Delta H)$ of each film was evaluated from the integrated intensity of the endothermic curve observed in the thermogram.

\section{RESULTS AND DISCUSSION}

\section{Overview of Behavior of HI-PVA with Different Heat Treatments}

Figure 1 shows dynamic storage Young's modulus $\left(E^{\prime}\right)$ and loss modulus $\left(E^{\prime \prime}\right)$ of HI-PVA films with $m m=0.78$ (the highest $\mathrm{mm}$ so far reported) at temperatures from $-50^{\circ} \mathrm{C}$ to $250^{\circ} \mathrm{C}$. The corresponding loss $\operatorname{tangent}(\tan \delta)$ is shown in Figure 2. The HI-PVA films examined here were annealed for $30 \mathrm{~min}$ at different temperatures, 40,120 , and $200{ }^{\circ} \mathrm{C}$, and subjected to the viscoelastic test with a heating mode. The degree of crystallinity $\chi_{c}$ of the film increased with an increase of the annealing temperature, as explained later in more detail.

The HI-PVA films exhibit four dispersions referred to as $\beta_{\mathrm{a}}, \alpha_{\mathrm{a}}, \beta_{\mathrm{c}}$ and $\alpha_{\mathrm{c}}$ in the order of increasing temperature, as most clearly noted for $\tan \delta$ (Figure 2). The major dispersion $\left(\alpha_{\mathrm{a}}\right)$ observed at around $70^{\circ} \mathrm{C}$ is attributable to the onset of micro-Brownian motion (i.e., glass transition) of the polymer chains in the 


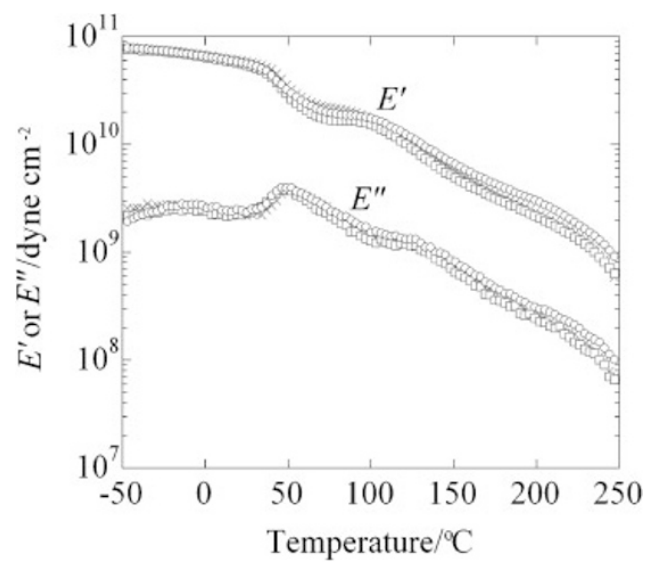

Figure 1. Temperature dependence of the dynamic storage Young's modulus $\left(E^{\prime}\right)$ and loss modulus $\left(E^{\prime \prime}\right)$ of HI-PVA films $(m m=0.78)$. The annealing temperature is $40^{\circ} \mathrm{C}$ (circle), $120^{\circ} \mathrm{C}$ (square) and $200^{\circ} \mathrm{C}$ (cross).

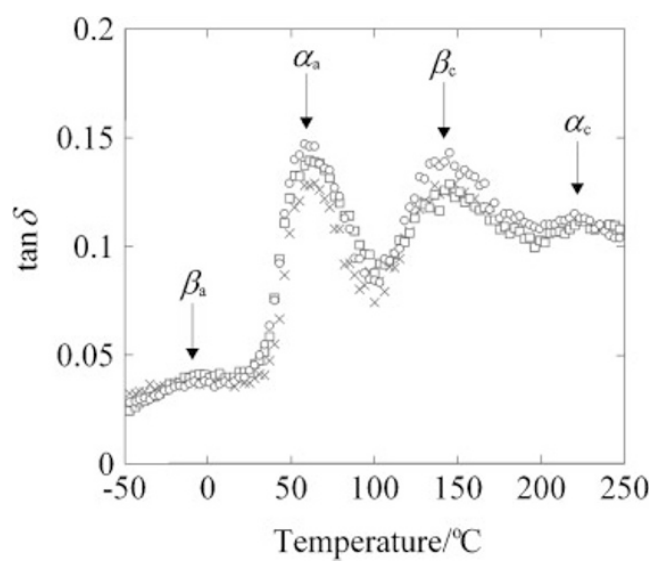

Figure 2. Temperature dependence of the loss tangent $(\tan \delta)$ of HI-PVA films $(m m=0.78)$. The annealing temperature is $40^{\circ} \mathrm{C}$ (circle), $120^{\circ} \mathrm{C}$ (square) and $200^{\circ} \mathrm{C}$ (cross).

non-crystalline region, as judged from the sharpness of the dispersion reflected in the large decrease of $E^{\prime}$ at around $70^{\circ} \mathrm{C}$. The $\beta_{\mathrm{a}}$ dispersion seen at around $-10^{\circ} \mathrm{C}$ can be assigned to the local twisting motion of non-crystalline chains. ${ }^{19}$ The $\alpha_{\mathrm{c}}$ and $\beta_{\mathrm{c}}$ dispersions, seen at around $140^{\circ} \mathrm{C}$ and $220^{\circ} \mathrm{C}$, occurs at temperatures well above the glass transition temperature and are attributable to the motion in the crystallites. ${ }^{20-23}$

The decrease of $E^{\prime}$ at around $70^{\circ} \mathrm{C}$ (Figure 1) can be used as a measure of the intensity of the $\alpha_{\mathrm{a}}$ dispersion (glass transition). This decrease becomes smaller on the increase of annealing temperature, which suggests that the content of the non-crystalline component decreases (and thus the degree of crystallinity $\chi_{c}$ increases) on sufficient annealing. (Indeed, the increase of $\chi_{\mathrm{c}}$ was confirmed, as explained later for Figure 3). At the same time, we note that the dispersion temperatures for all of the $\beta_{\mathrm{a}}, \alpha_{\mathrm{a}}, \beta_{\mathrm{c}}$ and $\alpha_{\mathrm{c}}$ processes hardly change with the annealing. Thus, the

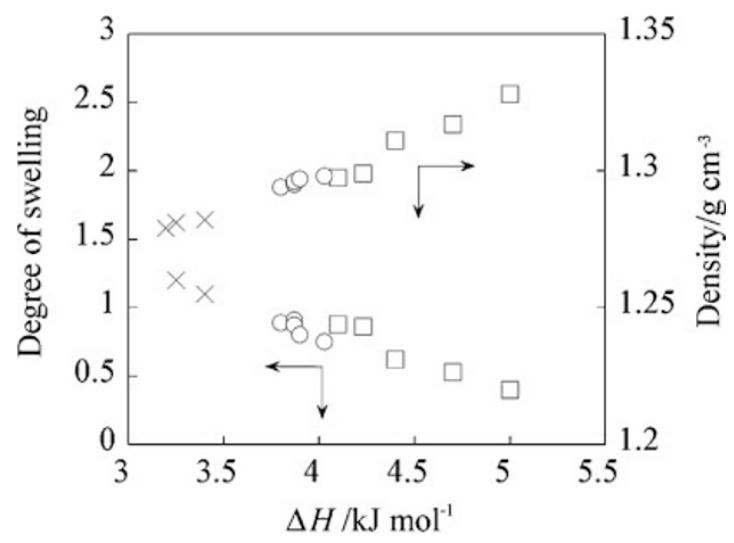

Figure 3. Dependence of the degree of swelling in water and the density on the $\Delta H$ values for HI-PVA $(m m=0.78$, circle), A-PVA $(m m=0.22$, cross $)$ and S-PVA $(m m=0.14$, square $)$.

mobility of respective HI-PVA chains in the crystalline and non-crystalline regions appears to be insensitive to the change in the degree of crystallinity induced by the annealing.

\section{Density, Swelling and Thermal Behavior of PVAs}

Since the viscoelastic behavior of PVA films changes not only with the stereoregularity but also with the crystallinity, the effect of stereoregularity on the behavior can be most clearly examined for films having the same crystallinity. The degree of crystallinity of PVA, $\chi_{\mathrm{c}}$, has been studied by various methods: X-ray diffraction, swelling, density and DSC measurement, etc. Among the various methods, the generally available is the DSC method, and $\chi_{\mathrm{c}}$ can be determined from the enthalpy change for melting, $\Delta H$. In this determination, the $\Delta H_{\mathrm{c}}$ value for the $100 \%$ crystallinity $\left(\Delta H_{\mathrm{c}}=6.87 \mathrm{~kJ} \mathrm{~mol}^{-1}\right)^{24}$ is often assumed to be independent of the stereoregularity. However, the crystal structure of HI-PVA differs from that of S-PVA and A-PVA, ${ }^{10}$ and no $\Delta H_{\mathrm{c}}$ data have been reported for HI-PVA.

For this problem, we here focus on the density $(\rho)$ and the degree of swelling $(Q)$ of HI-PVA, S-PVA, and A-PVA films in water. For HI-PVA $(m m=0.78)$, A-PVA $(m m=0.22)$ and S-PVA $(m m=0.14)$ films being annealed at several different temperatures and thus having different $\chi_{\mathrm{c}}$ values, we measured the $\rho$, $Q$ and $\Delta H$ values from the density, swelling and DSC measurements. In Figure 3, the $\rho$ and $Q$ values are plotted against the $\Delta H$. For the PVAs with different stereoregularities, $\rho$ and $Q$ exhibit a universal, almost linear dependence on $\Delta H$. These results suggest that the $\Delta H_{\mathrm{c}}$ value for the $100 \%$ crystallinity is almost same for the HI-PVA, S-PVA, and A-PVA films. In the absence of more detailed information for $\chi_{c}$, this paper utilizes the $\Delta H$ data and the $m m$-independent $\Delta H_{\mathrm{c}}$ value to estimate $\chi_{\mathrm{c}}$ of HI-PVA films. The width 


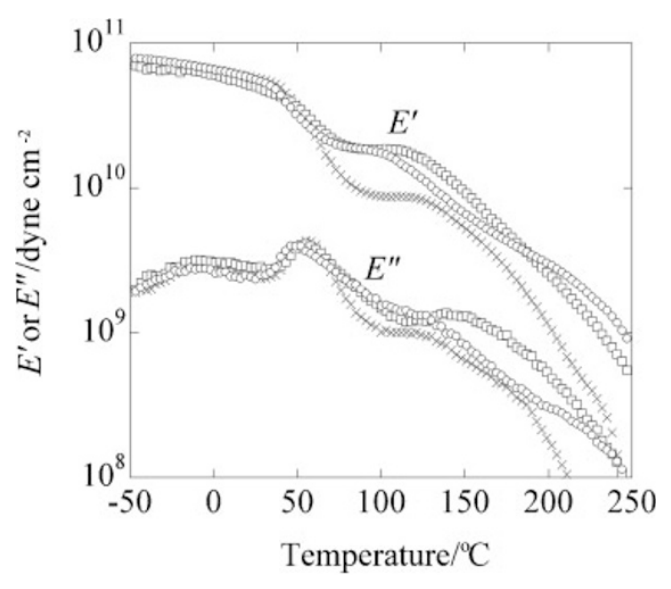

Figure 4. Temperature dependence of the dynamic storage Young's modulus $\left(E^{\prime}\right)$ and loss modulus $\left(E^{\prime \prime}\right)$ of HI-PVA $\left(\chi_{\mathrm{c}}=\right.$ 0.59 , circle), A-PVA $\left(\chi_{\mathrm{c}}=0.49\right.$, cross $)$ and S-PVA $\left(\chi_{\mathrm{c}}=0.59\right.$, square).

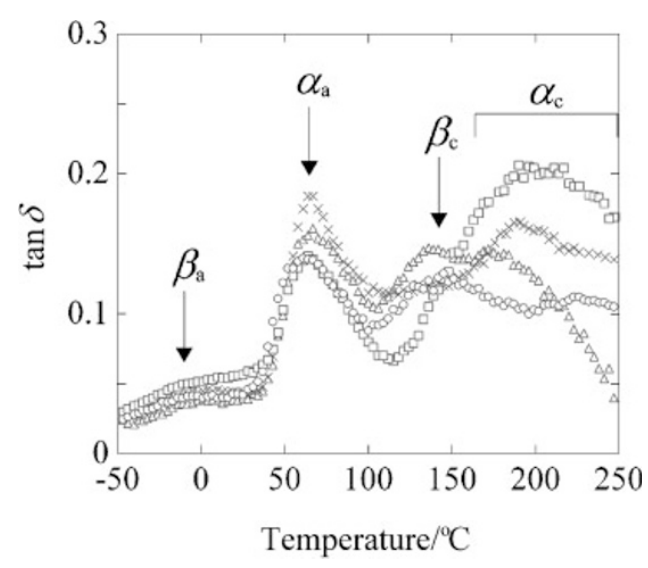

Figure 5. Temperature dependence of the loss tangent $(\tan \delta)$ of HI-PVA $\left(\chi_{\mathrm{c}}=0.59\right.$; circle $)$, LI-PVA $\left(\chi_{\mathrm{c}} \sim 0.3\right.$; triangle $)$, A-PVA $\left(\chi_{c}=0.49\right.$; cross $)$ and S-PVA $\left(\chi_{c}=0.59\right.$; square $)$.

of the scatter in the plots in Figure 3 suggests that the accuracy of this estimate is better than $\pm 10 \%$.

\section{Comparison of HI-PVA, S-PVA, and A-PVA Films}

Figure 4 shows a comparison of the temperature dependence of the $E^{\prime}$ and $E^{\prime \prime}$ for HI-PVA film $\left(\Delta H=4.03 \mathrm{~kJ} \mathrm{~cm}^{-1}\right.$; annealed at $\left.200^{\circ} \mathrm{C}\right)$, S-PVA film $\left(\Delta H=4.08 \mathrm{~kJ} \mathrm{~cm}^{-1}\right.$; annealed at $\left.40^{\circ} \mathrm{C}\right)$, and APVA film $\left(\Delta H=3.40 \mathrm{~kJ} \mathrm{~cm}^{-1}\right.$; annealed at $\left.200^{\circ} \mathrm{C}\right)$. The corresponding $\tan \delta$ is shown in Figure 5. The degree of crystallinity $\chi_{\mathrm{c}}$, determined satisfactorily from the $\Delta H$ value as explained in the previous section, is almost identical for the HI-PVA and S-PVA films $\left(\chi_{\mathrm{c}}=0.59\right)$ but somewhat smaller for the A-PVA film $\left(\chi_{c}=0.49\right)$. We can keep this point in our mind to relate the data in Figures 4 and 5 to the difference in the stereoregularity ( $\mathrm{mm}$ value), as discussed below.

As clearly seen in Figures 4 and 5, the $\beta_{\mathrm{a}}$ and $\alpha_{\mathrm{a}}$ dispersions occurring at around $-10^{\circ} \mathrm{C}$ and $70^{\circ} \mathrm{C}$

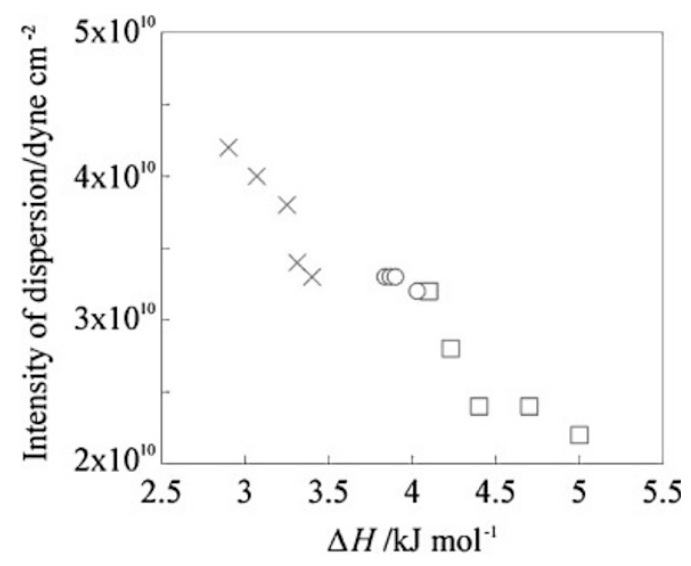

Figure 6. Dependence of the $\alpha_{\mathrm{a}}$ dispersion intensity measured as the decrease of $E^{\prime}$ on the degree of crystallinity estimated from $\Delta H$ for HI-PVA $(m m=0.78$; circle $)$, A-PVA $(m m=0.22 ;$ cross $)$ and S-PVA $(m m=0.14$; square).

are nearly the same, in both magnitude (reflected in the decrease of $E^{\prime}$ ) and location (noted as the peak temperature for $E^{\prime \prime}$ and/or $\tan \delta$ ), for the HI-PVA and S-PVA films having almost identical $\chi_{\mathrm{c}}$. For the A-PVA film having a smaller $\chi_{\mathrm{c}}$ value, the magnitude of the $\alpha_{\mathrm{a}}$ dispersion is larger but the location of the $\beta_{\mathrm{a}}$ and $\alpha_{\mathrm{a}}$ dispersions are the same compared to the HI-PVA and S-PVA films.

To clarify the effect of the stereoregularity on the non-crystalline chain motions of PVA, the $\tan \delta$ data for LI-PVA film ( $\mathrm{mm}=0.44$ and $\chi_{\mathrm{c}} \sim 0.3$; annealed at $200{ }^{\circ} \mathrm{C}$ ) are also shown in Figure 5. The degree of crystallinity of this LI-PVA film, obtained from a DSC measurement (only approximately because of its poor thermal stability at high temperatures ${ }^{14}$ ), was $\chi_{\mathrm{c}} \sim 0.3$. As seen in Figure 5, the local twisting motion (seen as the $\beta_{\mathrm{a}}$ dispersion) and micro-Brownian motion $\left(\alpha_{\mathrm{a}}\right.$ dispersion) of the respective chains in the non-crystalline region are essentially the same in the PVA films of various stereoregularities $(\mathrm{mm}=$ $0.14-0.78$ ). This behavior is in harmony with the change in the intensity of the $\alpha_{\mathrm{a}}$ dispersion (measured as the decrease of $E^{\prime}$ at around $70^{\circ} \mathrm{C}$ ) with $\Delta H$ (and $\chi_{\mathrm{c}}$ ) shown in Figure 6. The intensity appears to be almost universally dependent on $\Delta H$ (and $\chi_{\mathrm{c}}$ ) irrespective of the $\mathrm{mm}$ value, suggesting that the noncrystalline chain motion resulting in the $\alpha_{\mathrm{a}}$ dispersion is insensitive to the stereoregularity.

The $m m$-insensitivity of the $\alpha_{\mathrm{a}}$ and $\beta_{\mathrm{a}}$ dispersions is rather surprising because the stereoregularity is expected to affect the mobility of the non-crystalline chain. One possible origin of this insensitivity is the enrichment of the stereoregular sequences (either $m$ or $r$-sequences) of the PVA chains in the crystalline region and the corresponding reduction in the difference of the stereoregularities in the non-crystalline region of the samples. To examine this possibility, we 
followed the previous paper ${ }^{25}$ to apply the solid-state ${ }^{13} \mathrm{C}$ NMR analysis for the hydrated PVA films to estimate the mole fractions of the $m m, m r$, and $r r$ sequences for the mobile component that is produced by swelling with water. Our recent NMR experiment, ${ }^{26}$ however, revealed that the mole fractions of the respective sequences in the mobile component are very close to the mole fractions of the corresponding, overall triad tacticity for the HI-PVA and S-PVA films. These results indicate that the $m m, m r$, and $r r$ units should be almost equally distributed in the crystalline and non-crystalline regions for the HI-PVA and S-PVA films, which is in harmony with the result obtained for the A-PVA films. ${ }^{25}$ Thus, the chain motion in the non-crystalline region is insensitive to the stereoregularity therein, and an examination of the origin of this result requires more detailed solid-state ${ }^{13} \mathrm{C}$ NMR experiments. These experiments, including the detailed analysis of the $T_{1 \mathrm{C}}$ values of the non-crystalline components of the stereoregular PVA samples, are considered as an important subject of future work.

Now, we turn our attention to the dispersions of the crystalline components observed in Figures 4 and 5 at temperatures above $100^{\circ} \mathrm{C}$. The locations and intensities of these dispersions significantly change with the stereoregularity of the films. The HI-PVA sample $(m m=0.78)$ shows the major crystalline dispersion $\left(\beta_{\mathrm{c}}\right)$ at around $140^{\circ} \mathrm{C}$ and the side dispersion $\left(\alpha_{\mathrm{c}}\right)$ at around $220^{\circ} \mathrm{C}$. The LI-PVA sample $(\mathrm{mm}=0.44)$ shows the $\beta_{\mathrm{c}}$ dispersion at nearly the same temperature but its $\alpha_{\mathrm{c}}$ dispersion emerges at around $180^{\circ} \mathrm{C}$ (well below $T \alpha_{\mathrm{c}}$ for HI-PVA). For the A-PVA $(m m=0.22)$ and S-PVA $(m m=0.14)$ samples, no $\beta_{\mathrm{c}}$ dispersion is clearly detected and the $\alpha_{\mathrm{c}}$ dispersion emerges at around $195^{\circ} \mathrm{C}$ and $205^{\circ} \mathrm{C}$. These differences can be related to the structural origin(s) of the dispersions, as discussed below.

The $\beta_{\mathrm{c}}$ dispersion has been related to the transition in the expansion of the crystal $^{21}$ or a discontinuous point of the expansion coefficient of PVA. ${ }^{22}$ It resulted from a reduction of intermolecular forces in crystals, and this reduction takes place with thermal expansion of the PVA unit cells. ${ }^{19,21}$ As previously reported, successive intramolecular hydrogen bonding is formed for all $\mathrm{OH}$ groups along the chains in the crystalline region for the HI-PVAs. ${ }^{14}$ This perfect intramolecular bonding would in turn weaken the intermolecular bonding and thus enhance the sensitivity of the latter type of bonding to the unit cell expansion. This enhancement appears to result in the clear $\beta_{\mathrm{c}}$ dispersion seen for HI-PVA. For LI-PVA having a fairly large $\mathrm{mm}$ value $(=0.44)$, the $\beta_{\mathrm{c}}$ dispersion seems to be clearly resolved for the same reason. In contrast, for S-PVA, the $\beta_{\mathrm{c}}$ dispersion peak vanishes possibly because the strong intermolecular forces due to syndio- tactic regulation ${ }^{23}$ (and lack of significant intramolecular hydrogen bonding) hardly changes with the cell expansion. For A-PVA, the lack of the clear $\beta_{\mathrm{c}}$ dispersion peak can be also attributed to a similarly strong intermolecular forces as well as to high ordering in crystallites in A-PVA film, the latter resulting from the lamellar thickening on high-temperature annealing (i.e. at $200{ }^{\circ} \mathrm{C}$ ).

Differing from the $\beta_{\mathrm{c}}$ dispersion, the $\alpha_{\mathrm{c}}$ dispersion is assigned to thermal motion of the chain axis in the crystal lattices. ${ }^{22,23}$ Figure 5 clearly indicates that the $\alpha_{\mathrm{c}}$ dispersion temperature $T \alpha_{\mathrm{c}}$ decreases in the order of HI-PVA $\left(220^{\circ} \mathrm{C}\right)>$ S-PVA $\left(205^{\circ} \mathrm{C}\right)>$ APVA $\left(195^{\circ} \mathrm{C}\right)>$ LI-PVA $\left(180^{\circ} \mathrm{C}\right)$. This order, well corresponding to the order of the other physical properties, $T_{\mathrm{m}}, \chi_{\mathrm{c}}$, and $T_{1 \mathrm{C}}$, is not in accord to the order of the $m m$ value. In other words, plots of $T \alpha_{\mathrm{c}}, T_{\mathrm{m}}, \chi_{\mathrm{c}}$, and $T_{1 \mathrm{C}}$ against $\mathrm{mm}$ show minima at $\mathrm{mm}=0.4-0.5$ (for LI-PVA). This behavior of the physical properties including $T \alpha_{\mathrm{c}}$ suggests that the disordering of the intermolecular and/or intramolecular hydrogen bonding along each PVA chain, being most significant at $m m=0.4-0.5$, governs these properties. ${ }^{14}$ In particular, the enhancement of the intermolecular bonding on a decrease of $\mathrm{mm}$ below 0.4 (for A-PVA and S-PVA) would naturally reduce the axial mobility of respective chains in the crystal lattice, while the enhancement of intramolecular bonding on an increase of $\mathrm{mm}$ above 0.5 (for HI-PVA) would stiffen the chain to reduce the axial mobility. The reduction of the mobility due to these two types of enhancement results in an increase of $T \alpha_{\mathrm{c}}$ thereby possibly giving the order of $T \alpha_{c}$ explained above.

\section{CONCLUSION}

We have examined dynamic viscoelasticity of highly isotactic poly(vinyl alcohol) (HI-PVA, $m m=0.78$ ) in comparison with syndiotactic PVA (S-PVA, $m m=$ 0.14 ) and atactic PVA (A-PVA, $m m=0.22$ ). The relaxation in the non-crystalline region, the $b_{\mathrm{a}}$ and $a_{\mathrm{a}}$ dispersions reflecting the local twisting motion and micro-Brownian motion of the chains in this region, were nearly the same, in both magnitude and location, for the HI-PVA and S-PVA having an almost identical degree of crystallinity. Our recent solid-state ${ }^{13} \mathrm{C}$ NMR experiments revealed that there is no enrichment of the stereoregular sequences (neither $m$ or $r$-units) of the PVA chains in the crystalline region for each PVA sample. To clarify the origin of this surprising result, the insensitivity of the chain motion in the non-crystalline region to the stereoregularity, more detailed solid-state ${ }^{13} \mathrm{C}$ NMR experiments will be made in future.

In contrast, the $\beta_{\mathrm{c}}$ and $\alpha_{\mathrm{c}}$ dispersions in the crystal- 
line region changed with the stereoregularity. The $\beta_{\mathrm{c}}$ dispersion (local motion in crystals due to defects) was clearly observed for HI-PVA but not for S-PVA and A-PVA. The $\alpha_{\mathrm{c}}$ dispersion (axial motion of the chain in the crystal lattices) was observed for all PVA films but its temperature increased in the order of HI-PVA > S-PVA > A-PVA, which well corresponded to the order of the melting temperature or ${ }^{13} \mathrm{C}$ spin-lattice relaxation time of these PVAs. These results may be related to a difference of the magnitude of intermolecular and/or intramolecular hydrogen bonding in the crystals of respective PVAs. Specifically, the successive intramolecular bonding in the HIPVA crystal appears to reduce the magnitude of the intermolecular bonding and stiffen the chain backbone, thereby allowing the chain to exhibit the $\beta_{\mathrm{c}}$ motion and increase the $\alpha_{\mathrm{c}}$ dispersion temperature.

\section{REFERENCES}

1. K. Fujii, J. Polym. Sci., Part D, 5, 431 (1971).

2. "Polyvinyl Alcohol-Developments," C. A. Finch, Ed., John Wiley \& Sons, New York, 1992, chap. 9 and 10.

3. "Polyvinyl Alcohol," C. A. Finch, Ed., John Wiley \& Sons, New York, 1973, chap. 6 and 10.

4. S. Murahasi, H. Yuki, T. Sano, U. Yonemura, T. Tadokoro, and Y. Chatani, J. Polym. Sci., S77, 62 (1962).

5. H. Yuki, K. Hatada, K. Oda, H. Kinoshita, S. Murahashi, K. Ono, and Y. Ito, J. Polym. Sci., Part A-1, 7, 1517 (1969).

6. S. Okamura, T. Kodama, and T. Higashimura, Makromol. Chem., 53, 180 (1962).

7. T. Higashimura, K. Suzuki, and S. Okamura, Makromol.
Chem., 86, 259 (1965).

8. S. Murahashi, S. Nozakura, and M. Sumi, J. Polym. Sci. Part B, 3, 245 (1965).

9. a) S. Murahashi, S. Nozakura, M. Sumi, H. Yuki, and K. Hatada, J. Polym. Sci., Polym. Lett. Ed., 4, 65 (1966).

b) Kobunshi Kagaku, 23, 550 (1966).

10. H. Ohgi and T. Sato, Macromolecules, 26, 559 (1993).

11. H. Ohgi and T. Sato, Macromolecules, 32, 2403 (1999).

12. H. Ohgi and T. Sato, Polymer, 43, 3829 (2002).

13. F. Horii, S. Hu, K. Deguchi, H. Sugisawa, H. Ohgi, and T. Sato, Macromolecules, 29, 3330 (1996).

14. H. Ohgi, T. Sato, S. Hu, and F. Horii, Polymer, 47, 1324 (2006).

15. A. Nagai and M. Takayanagi, Kogyo Kagaku Zassi, 68, 96 (1965).

16. T. Yamamoto, S. Yoda, H. Takase, O. Sangen, R. Fukae, M. Kamachi, and T. Sato, Polym. J., 23, 185 (1991).

17. T. Moritani, I. Kuruma, K. Shibatani, and Y. Fujiwara, Macromolecules, 5, 577 (1972).

18. J. R. DeMember, H. C. Haas, and R. L. MacDonald, J. Polym. Sci., Polym. Lett. Ed., 10, 385 (1972).

19. A. Nagai and M. Takayanagi, Kogyo Kagaku Zasshi, 68, 836 (1965).

20. K. Sugiura, M. Hashimoto, S. Matsuzawa, and K. Yamaura, J. Appl. Polym. Sci., 82, 1291 (2001).

21. K. Shiraishi, K. Ishikawa, and K. Miyasaka, Kobunshi Kagaku, 21, 588 (1964).

22. T. Yano, Busseiron Kenkyu, 94, 176 (1956).

23. R. Fukae, T. Yamamoto, H. Masago, N. Kawatsuki, O. Sangen, and M. Kamachi, Sen'i Gakkaishi, 53, 195 (1997).

24. R. K. Tubbs, J. Polym. Sci., 3, 4181 (1965).

25. K. Masuda, H. Kaji, and F. Horii, Polym. J., 33, 356 (2001).

26. H. Ohgi, H. Yang, T. Sato, and F. Horii, to be published. 